\title{
Cómo afrontan los adolescentes mexicanos el malestar depresivo
}

Depression coping strategies in Mexican adolescents

\author{
Sergio González Escobar \\ José Luis Valdez Medina \\ Norma Ivonne González-Arratia López-Fuentes \\ Dulce Belén Velasco Monroy \\ Universidad Autónoma del Estado de México
}

\begin{abstract}
Resumen
Los adolescentes son una población de alto riesgo de padecer depresión, observándose que es cada vez a edades más tempranas cuando se presenta, lo que hace que quienes la padecen en esta etapa de la vida la sufran hasta por dos años antes de buscar ayuda. Asimismo, las investigaciones en México han descuidado el estudio de las estrategias que los adolescentes emplean para afrontar la depresión. Por ello, el objetivo de la presente investigación fue identificar las estrategias que los adolescentes emplean cuando se sienten deprimidos. Para ello se trabajo con una muestra no probabilística conformada por 400 adolescentes, la mitad con escolaridad del nivel medio básico y el resto en el nivel medio superior. Asimismo, estuvieron repartidos equitativamente por sexo. Se utilizó la técnica de redes semánticas Naturales y todas las aplicaciones se realizaron dentro de los salones de clase. Los resultados mostraron que hombres y mujeres coinciden de manera importante por nivel educativo en las definidoras proporcionadas pero cuando se agrupan los hombres y las mujeres, por muestras totales, ya no coinciden tanto. De igual manera, se considera que la técnica resulta adecuada para el estudio. Por último se plantea que las estrategias empleadas por los adolescentes en realidad no les ayudan a disminuir las manifestaciones de la depresión y sólo funcionan como paliativos.
\end{abstract}

Palabras clave: Depresión, adolescentes, estrategias, afrontamiento.

Nota del autor:

Sergio González Escobar, Centro Universitario, Universidad Autónoma del Estado de México; José Luis Valdez Medina, Facultad de Psicología, Universidad Autónoma del Estado de México; Norma Ivonne González-Arratia López-Fuentes, Facultad de Psicología, Universidad Autónoma del Estado de México; Dulce Belén Velasco Monroy, Centro Universitario, Universidad Autónoma del Estado de México.

La correspondencia en relación con este artículo debe dirigirse a Sergio González Escobar, Centro Universitario, Universidad Autónoma del Estado de México, Atlacomulco, Km 60, Carretera Libre Toluca-Atlacomulco, C.P. 50455, Atlacomulco, Estado de México, México.

Correo electrónico: sergioglz4@ hotmail.com 


\section{Abstract}

In regard to depression, adolescents are among the highest at-risk members of the population and research findings reveal that the onset age of this disease is descending. Consequently those who suffer from depression in this age period usually wait around two years before asking for attention. In addition, Mexican research has neglected the study of the coping strategies that adolescents use when they are depressed. Such a study is the aim of the here reported investigation. A non-probabilistic sample of 400 adolescents attending elementary and high schools was investigated. Half of them were boys and the rest were girls. The Natural Semantic Network was used as instrument, which was administered at students' classrooms. Results showed that students consistently define their strategies across educational level; yet such a consistency is not present when boys and girls are grouped by total samples. The use of the Natural Semantic Network was appropriate for the intended goal of the study. An important finding is that the coping strategies that adolescents use are not helpful in achieving healthy states and in preventing the occurrence of depression, working only as mere palliatives.

Keywords: Depression, adolescents, coping strategies.

El número aproximado de adolescentes y jóvenes en el planeta es de 1700 millones; representan una cuarta parte de la población mundial. En México, los datos referentes a las condiciones socioeconómicas que describen a este grupo de edad, en particular, no son alentadores, ya que de acuerdo con la información proporcionada por el Instituto Nacional de Estadística, Geografía e Informática (2000) se estima que un poco más del 21 por ciento de la población se encuentra entre la adolescencia y la juventud, lo que representa cerca de 30 millones de mexicanos. De éstos, entre el 35 y 40 por ciento viven en situaciones de extrema pobreza; al llegar a los 19 años, cerca del 89 por ciento abandonan la escuela.

Su participación en el mercado laboral es importante, el 8 por ciento de los adolescentes que tienen entre 12 y 14 años se encuentra trabajando, cifra que asciende hasta el 35 por ciento cuando las edades oscilan entre los 15 y 19 años de edad.

Respecto a su vida sexualmente activa, se observa que ésta inicia alrededor de los 15 años; en el área rural es a los 13.8 años; mientras que en la urbana comienza en promedio a los 16.7 años (CONAPO, 2002; Encuesta Nacional de la Juventud, 2000; Santos-Preciado, Villa-Barragán, García-Aviles, León-Álvarez, Quezada-Bolaños, \& Tapia-Conyer, 2003).
Distintos autores (Craig, 1997; Shaffer, 2000; Papalia, 2004), han considerado que la adolescencia es uno de los periodos más difíciles de la vida, en la que los cambios físicos, psicológicos y sociales, asociados a condiciones mundiales y nacionales de crisis económicas, así como las escasas oportunidades educativas y laborales; impactan en la salud mental de los adolescentes.

Por consiguiente, el Consejo Nacional Contra las Adicciones (2000) ha señalado que uno de los trastornos que más aqueja a esta parte de la población es la depresión. De acuerdo con González, Cruz y Martínez (2007) en los adolescentes, a diferencia de los adultos, se caracteriza por sentimientos de tristeza, soledad, enojo, angustia, irritabilidad. A nivel cognitivo tienen dificultades para concentrarse, así como para tomar decisiones; fisiológicamente, tienden más al Ilanto, se alteran los patrones de sueño y de alimentación, presentando letargo y fatiga; a nivel conductual, la característica principal es el aislamiento social, incluso de sus amigos que, después de la familia, se convierte en el vínculo social más importante para el adolescente. Estos autores señalan que existen diferencias en las manifestaciones entre los y las adolescentes; en el caso de los varones se observa mayor aislamiento y la apreciación subjetiva de hablar menos de lo normal, mientras que las mujeres presentan mayores sentimientos de soledad. 
A partir de los estudios realizados, respecto a la depresión en este grupo, se ha observado que, al igual que en los adultos, se presenta en dos mujeres por cada hombre (Mariño, MedinaMora, Chaparro, \& González-Forteza, 1993); la edad promedio en que aparece por primera vez es a los 17 años (Medina-Mora \& cols, 2003); la cantidad de adolescentes que presentan síntomas depresivos oscila entre el 22 y el 50 por ciento en población abierta (Boschetti-Fentanes, 2004), en México corresponde aproximadamente a dos millones de adolescentes (Benjet, Borges, Medina-Mora, Fleiz-Bautista, \& Zambrano-Ruiz, 2004); y el 50 por ciento de quienes llegan a presentarla pueden sufrir hasta por dos años antes de recibir la atención adecuada, debido principalmente a la falta de conocimiento del tratamiento (Cevallos-Rivera, Ochoa-Muñoz, \& Cortez-Pérez, 2000).

Estos periodos tan prolongados que sufre el adolescente con la depresión, antes de tener la atención adecuada, permiten pensar en la forma en que la están afrontando; ya que, si bien es cierto que no reciben ayuda, es posible que estén utilizando estrategias para controlar los sentimientos y pensamientos que genera. Sin embargo, se entiende como afrontamiento a los esfuerzos cognoscitivos y conductuales en constante cambio, que se desarrollan en respuesta a las demandas específicas internas o externas evaluadas como excedentes 0 desbordantes de los propios recursos personales (Lazarus \& Folkman, 1986). El afrontamiento ha sido poco estudiado en México, sobre todo en su vínculo con la depresión, a pesar de que se ha observado que es un elemento clave que incide en las condiciones emocionales de los adolescentes (González-Forteza, Villatoro, Pick, \& Collado, 1998).

De esta forma, las investigaciones encaminadas al estudio de los estilos de afrontamiento versan sobre las estrategias que emplean los adolescentes ante estresores familiares y psicosociales (González-Forteza, 1992; González-Forteza, Pick, Collado, Álvarez, \& Jiménez, 1996; González-Forteza, AndradePalos, \& Jiménez, 1997; González-Forteza et al., 1998). Los resultados mostraron que cuando los adolescentes presentaban algún estresor social o familiar, las estrategias que comúnmente se empleaban eran: resolutivas, adaptativas, de distracción, de agresión manifiesta o encubierta, evitativas, reflexivas, así como expresiones de ánimo depresivo; siendo las mujeres las que marcaban las diferencias por sexo.

En un estudio, empleando una muestra argentina, se observó que cuando los adolescentes afrontaban los problemas lo hacían a través de la preocupación, buscar diversiones relajantes, fijarse en lo positivo, buscar pertinencia y la distracción física (Figueroa, Contini, Lacuzana, Levín, \& Estévez, 2005); mientras que los adolescentes norteamericanos empleaban las estrategias de buscar apoyo social, espiritual y profesional, concentrarse en resolver el problema, esforzarse y tener éxito, preocuparse, invertir en amigos íntimos, buscar pertenencia, hacerse ilusiones, falta de afrontamiento, reducción de la tensión, acción social, ignorar el problema, autoinculparse, reservarlo para sí, fijarse en lo positivo, buscar diversiones relajantes y la distracción física (Frydenberg \& Lewis, 1997) .

La investigación realizada por Meyer (2007), en la que empleó el Coping Inventory of Depression, y lo aplicó a estudiantes universitarios de una institución privada, encontró que éstos afrontaban sus sentimientos de tristeza al salir y realizar actividades sociales, expresar sus sentimientos y la distracción intencional. Asimismo, observó que había estrategias que se usaban más de lo que servían; tales como el expresar sus sentimientos, la distracción o ignorar el problema. En cambio, las estrategias que se usaban menos, pero que tenían mejores efectos, eran las actividades recreativas, buscar el significado, actividades de utilidad, rezar y buscar ayuda de los expertos.

Es conveniente señalar que esta investigación presenta varios problemas en su diseño, los dos más importantes son el haber empleado una muestra heterogénea y no representativa de estudiantes de psicología. Por otro lado, el instrumento, si bien es cierto que se ha aplicado en muestras españolas, en México no existen estudios respecto a sus propiedades psicométricas que justifiquen su uso. Siguiendo esta misma línea de investigación Galaif, Sussman, Chou y Wills (2003) mencionan que la búsqueda de apoyo social se relaciona con los estilos de afrontamiento ante la tristeza. 
Los estudios que han vinculado la depresión con los estilos de afrontamiento se han originado en contextos distintos a la población mexicana, donde los resultados mostraron que cuando las personas se comienzan a deprimir emplean estrategias de manera espontánea sin saber si les van a ser de utilidad (Ring \& Vázquez, 1996). Las primeras en utilizarse son de tipo cognitivo, siempre y cuando exista la posibilidad de controlar las emociones, cuando esto ya no es posible lo afrontan desde lo emocional (Ando, 2002). Asimismo, se ha observado que a mayor uso de estilos de afrontamiento orientados a la solución del problema y en resignificar positivamente, existe menor dolor emocional, menos síntomas depresivos y mayor bienestar; mientras que el afrontamiento, orientado a la emoción, pasivo y con tendencia al actino-out se correlaciona con mayor dolor emocional y depresión (Kuyken \& Brewin, 1994; Haghitgou \& Peterson, 2001; McWilliams, Cox, \& Enns, 2003). Aunado a lo anterior, Marusic y Goodwin (2006), así como Sheung-tak y Chan (2007), han señalado que el empleo de estrategias maladaptativas de afrontamiento y las dificultades interpersonales son variables asociadas a la depresión y a la conducta suicida.

Cuando la depresión incrementa su intensidad se observa que las estrategias empleadas difícilmente podrán resolver este trastorno, ya que están orientadas a distraerse del problema y las que más se utilizan son las de tipo emocional y pasivo (Whatley, Foreman, \& Richards, 1998), el optimismo ilusorio (Wishful thinking), la rumiación y la búsqueda de información compulsiva sobre su situación (Smith, Wethington, \& Zhan, 1996); así como la distracción y la represión (Ring \& Vázquez, 1996).

Respecto al género, se ha observado que las mujeres, a diferencia de los hombres, tienden a emplear más estrategias que se centran en la distracción (Hewitt, Flett, \& Endler, 1995), el escape-evitación, el autocontrol y el aceptar la responsabilidad de la situación (Kuyken \& Brewin, 1994).

Particularmente en México, Gamboa y Flores (2006), teniendo como objetivo determinar la relación entre la asertividad, el enfrentamiento y la depresión en adolescentes hombres y mujeres, realizaron una investigación en la que sus resultados mostraron que los adolescentes que eran asertivos se expresaban y enfrentaban los problemas de manera directa, presentaban menores índices de depresión; y que a mayor asertividad indirecta y no asertividad la sintomatología depresiva era más alta. Asimismo, se observó que a mayor depresión mayor uso de los estilos emocional evasivo y emocional negativo; por el contrario, a menor depresión los estilos más utilizados eran el directo, el revalorativo y el directo revalorativo.

Como se habrá podido observar, son pocos los estudios que se han realizado para comprender cómo es que los adolescentes afrontan la depresión. A pesar de los resultados de otros países, no se pueden trasladar a contextos como el de los adolescentes mexicanos, ya que como lo señalan Hussain y Cochrane (2003), las diferencias culturales son un factor que influye en la elección de las estrategias a emplear. Un claro ejemplo de ello, el que las mujeres en la India eligen sus estrategias en función de sus creencias (rezan y tienen fe en que Dios sabe lo que hace). De esta forma, el objetivo de la presente investigación fue identificar cuáles son las estrategias que emplean adolescentes mexicanos cuando consideran estar deprimidos.

\section{Método}

\section{Participantes}

Se trabajó con una muestra no probabilística de tipo intencional conformada por 400 estudiantes (200 del nivel Medio Básico y 200 del Medio Superior). Repartidos equitativamente por sexo y cuyas edades oscilaron entre los 12 y 20 años de edad, con una media $=15.16$ (D.E. $=1.8)$.

\section{Instrumento}

Como instrumento se empleó la técnica de Redes Semánticas Naturales (Figueroa, González, \& Solís, 1981; Valdez-Medina \& Hernández, 1986), que consiste fundamentalmente en dos tareas sencillas: 
Definir una palabra estímulo con un mínimo de cinco palabras sueltas que pueden ser verbos, adverbios, sustantivos, adjetivos, pronombres; sin utilizar ninguna partícula gramatical (artículos o preposiciones).

Jerarquizar todas y cada una de las palabras que dieron como definidoras, en función de la importancia que consideraron que tienen para con la palabra estímulo, asignándole el número uno a la palabra más cercana, relacionada o que mejor defina la palabra estímulo, el número dos a la que le sigue en relación, el tres a la siguiente, y así sucesivamente hasta terminar de jerarquizar todas las palabras definidoras que generaron.

Por el propósito de la investigación el único estímulo empleado fue: "¿Qué haces o has hecho para dejar de estar deprimido?"

\section{Procedimiento}

Todas las aplicaciones se realizaron de manera grupal dentro de los salones de clase. Se les explicó el objetivo de la investigación e invito a participar en forma voluntaria, cabe mencionar que ningún adolescente se negó a hacerlo.

Los valores obtenidos fueron el Conjunto SAM, que consiste en las 15 definidoras con valor $M$ más alto; el valor $\mathrm{M}$ total, que consiste en el peso semántico de las definidoras; así como el valor $\mathrm{J}$, que consiste en el número total de definidoras proporcionadas por los participantes.

\section{Resultados}

Los resultados obtenidos por el estímulo "¿Qué haces o has hecho para dejar de estar deprimido?", mostraron que los hombres y las mujeres estudiantes del Nivel Medio Básico coinciden en el 80 por ciento de las definidoras proporcionadas. Mientras que los hombres emplearon las definidoras dibujar, video juegos y deportes, las mujeres emplearon leer, caminar y hablar. El valor $\mathrm{J}$ más alto lo presentan los varones (véase tabla 1).
En el Nivel Medio Superior, el conjunto SAM obtenido del estímulo ¿Qué haces o has hecho para dejar de estar deprimido? por hombres y mujeres, mostró que coincidieron en el 80 por ciento de las definidoras proporcionadas. Los hombres emplearon las definidoras dibujo, ayuda y ver; mientras que las mujeres emplearon amigos, deporte y cantar. Nuevamente el valor $\mathrm{J}$ fue más alto en hombres (véase tabla 2).

El conjunto SAM del estímulo "¿Qué haces o has hecho para dejar de estar deprimido?" de la muestra total por hombres y mujeres, mostraron una coincidencia del 40 por ciento y se diferenciaron en el 60 por ciento de las definidoras empleadas. Los hombres utilizaron jugar, platicar, escuchar música, televisión, leer, salir, reír, pensar y pasear; mientras que las mujeres emplearon amigos, familia, feliz, deporte, bailar, cantar, gritar, escribir y positivismo. El valor $\mathrm{J}$, al igual que en los casos anteriores, es más alta en hombres (véase tabla 3).

Los resultados obtenidos del conjunto SAM por nivel educativo del estímulo "¿Qué haces o has hecho para dejar de estar deprimido?", mostraron una coincidencia del 80 por ciento y una diferencia del 20 por ciento. De esta forma, mientras que los adolescentes de secundaria corren, dibujan y pasean; los de educación media superior olvidan, cantan y van a fiestas (véase tabla 4).

\section{Discusión}

Como se ha podido observar a lo largo de la presente investigación, el estudio de la depresión en adolescentes se ha orientado a determinar su relación con otras variables y muy poco sobre la forma en que éstos la afrontan, sobre todo en el contexto nacional. En este sentido, el objetivo de la presente investigación fue obtener, a partir del uso de la técnica de Redes Semánticas Naturales, las estrategias que los adolescentes emplean. Cabe señalar que, la técnica permitió obtener información precisa respecto a este tema y continúa demostrando su utilidad como una etnometodología adecuada para el estudio de los significados, orientando el estudio desde una perspectiva etnopsicológica y apta, no sólo para la investigación en psicología social (ValdezMedina, 1998), sino también en la psicología clínica (González \& Valdez, 2005). 
Tabla 1

Conjunto SAM del Estimulo ¿Qué haces o has hecho para dejar de estar deprimido?, en estudiantes de Nivel Medio B

\begin{tabular}{lllll}
\hline \multicolumn{2}{c}{ CVO. } & HOMBRES & \multicolumn{3}{c}{ MUJERES } \\
& DEFINIDORAS & VM & DEFINIDORAS & VM \\
\hline 1 & Jugar & 389 & Jugar & 357 \\
2 & Platicar & 230 & Salir & 316 \\
3 & Televisión & 194 & Oír música & 303 \\
4 & Salir & 185 & Platicar & 297 \\
5 & Oír música & 183 & Televisión & 211 \\
6 & Correr & 159 & Amigos(as) & 167 \\
7 & Dibujar & 145 & Leer & 157 \\
8 & Dormir & 132 & Reír & 134 \\
9 & Video juegos & 128 & Dormir & 116 \\
10 & Amigos(as) & 108 & Correr & 114 \\
11 & Pensar & 107 & Comer & 108 \\
12 & Comer & 102 & Caminar & 99 \\
13 & Pasear & 96 & Pensar & 97 \\
14 & Reír & 94 & Hablar & 95 \\
15 & Deportes & 93 & Pasear & 95 \\
& Valor J = 199 & & Valor J = 132 & \\
\hline
\end{tabular}

Tabla 2

Conjunto SAM del Estimulo ¿Qué haces o has hecho para dejar de estar deprimido?, por hombres y mujeres de Nivel Medio Superior

\begin{tabular}{lllll}
\hline CVO. & HOMBRES & & MUJERES & \\
& DEFINIDORAS & VM & DEFINIDORAS & VM \\
\hline 1 & Jugar & 453 & Música & 442 \\
2 & Platicar & 382 & Salir & 439 \\
3 & Música & 283 & Platicar & 393 \\
4 & Dibujo & $\mathbf{2 6 7}$ & Jugar & 315 \\
5 & Televisión & 247 & Televisión & 206 \\
6 & Salir & 247 & Dormir & 196 \\
7 & Leer & 225 & Leer & 190 \\
8 & Dormir & 206 & Caminar & 184 \\
9 & Pensar & 167 & Pensar & 184 \\
10 & Ayuda & $\mathbf{1 5 1}$ & Reír & 184 \\
11 & Reír & 150 & Comer & 131 \\
12 & Caminar & 135 & Amigos & $\mathbf{1 2 6}$ \\
13 & Distraerme & 130 & Distraerme & 115 \\
14 & Comer & 126 & Deporte & 101 \\
15 & Ver & $\mathbf{1 1 9}$ & Cantar & 96 \\
& J = 127 & & J = 94 & \\
\hline
\end{tabular}


Tabla 3

Conjunto SAM del Estimulo ¿Qué haces o has hecho para dejar de estar deprimido?, por hombres y mujeres

\begin{tabular}{lllll}
\hline CVO. & HOMBRES & & MUJERES & \\
& DEFINIDORAS & VM & DEFINIDORAS & VM \\
\hline 1 & Jugar & $\mathbf{9 4 8}$ & Dormir & 307 \\
2 & Platicar & $\mathbf{6 2 8}$ & Amigos & $\mathbf{2 9 0}$ \\
3 & Escuchar Música & $\mathbf{5 9 5}$ & Caminar & 283 \\
4 & Televisión & $\mathbf{5 1 1}$ & Comer & 269 \\
5 & Dormir & 394 & Familia & $\mathbf{2 0 2}$ \\
6 & Dibujar & 378 & Correr & 180 \\
7 & Leer & $\mathbf{3 3 0}$ & Feliz & 180 \\
8 & Salir & $\mathbf{3 2 5}$ & Deporte & 174 \\
9 & Correr & 312 & Distraerme & 171 \\
10 & Comer & 258 & Bailar & 153 \\
11 & Reír & $\mathbf{2 5 1}$ & Cantar & 152 \\
12 & Pensar & $\mathbf{2 4 7}$ & Gritar & $\mathbf{1 2 6}$ \\
13 & Pasear & $\mathbf{2 4 3}$ & Escribir & $\mathbf{1 2 0}$ \\
14 & Caminar & 229 & Positivismo & 112 \\
15 & Distraerme & 211 & Dibujar & 108 \\
& J =173 & & J =94 & \\
\hline
\end{tabular}

Tabla 4

Conjunto SAM del Estímulo ¿Qué haces o has hecho para dejar de estar deprimido? por nivel educativo

\begin{tabular}{lllll}
\hline CVO. & SECUNDARIA & & PREPARATORIA & DEFINIDORAS \\
& DEFINIDORAS & VM & VM \\
\hline 1 & Jugar & 746 & Jugar & 866 \\
2 & Platicar & 622 & Platicar & 775 \\
3 & Salir & 501 & Escuchar música & 725 \\
4 & Escuchar Música & 486 & Salir & 686 \\
5 & Televisión & 405 & Televisión & 443 \\
6 & Correr & 273 & Pensar & 428 \\
7 & Dormir & 248 & Leer & 415 \\
8 & Reír & 228 & Dormir & 402 \\
9 & Leer & 227 & Reír & 334 \\
10 & Dibujar & 221 & Caminar & 319 \\
11 & Comer & 210 & Comer & 309 \\
12 & Pensar & 204 & Deportes & 200 \\
13 & Pasear & 191 & Olvidar & 177 \\
14 & Caminar & 176 & Cantar & 164 \\
15 & Deportes & 144 & Fiestas & 163 \\
& J 331 & & J = 221 & \\
\hline
\end{tabular}


Los resultados obtenidos por sexo mostraron que en México, a pesar de que en la actualidad los hombres y las mujeres se están tornando andróginos (Valdez, 2007), a los primeros se les ha enseñado que no deben de ser sentimentales; mientras que a las segundas se les permite cualquier manifestación de sus emociones. Así, los resultados muestran la condición instrumental de los hombres, donde se muestra que, al no poder expresar sus emociones abiertamente a través del llanto o la tristeza, tienen que emplear otras vías de expresión aceptadas socialmente; por ello recurren a otro tipo de medios para dejar de estar deprimidos. En este caso, las estrategias están orientadas al hacer, al impulso, al movimiento, tales como el jugar, salir o pasear. Esto mismo permite explicar el porque los hombres presentaron valores más altos. En cambio, las mujeres están orientadas a la expresión, son más pasivas y, al ser más expresivas, tienen mayores posibilidades de demostrar sus emociones y no existe ningún inconveniente en que lloren o estén tristes, por ello son tan consistentes al momento de utilizar sus estrategias.

Asimismo, los resultados mostraron que existen estrategias que se usan de manera constante, por lo menos durante estos seis años que abarcan los dos niveles educativos estudiados. A la primera de ellas, se les puede llamar Estrategias de Regulación Fisiológica, debido a que los adolescentes afrontan la depresión a través del comer y el dormir, de esta manera disminuyen la ansiedad y evitan, aunque sea por un momento el dolor, que implica. Esto lo consiguen a través de evitar el contacto con el mundo y con la fantasía de que cuando despierten todo será distinto. Estas conductas, como estrategias de afrontamiento, son sumamente importantes, debido a que tradicionalmente se les ha considerado como los síntomas biológicos prototípicos de la depresión (Pardo, Sandoval, \& Umbarila, 2004). En cambio, los resultados aquí encontrados los muestran como conductas que tienen para disminuir el malestar que implica.

Un segundo tipo de estrategias, mencionada por los adolescentes, se le puede llamar Estrategias de Regulación Física, debido a que el adolescente corre, camina, hace ejercicio o practica algún deporte. En este caso, lo que se pretende es expulsar el malestar del cuerpo a través de una actividad física. Sin embargo, a pesar de que se ha mencionado que el hacer ejercicio mejora la salud física y mental, habrá que estudiar el momento en que se recomienda hacerlo y considerarlo como una estrategia adecuada para disminuir la depresión (CevallosRivera et al., 2000).

Otro tipo de estrategias, que han sido planteadas por diversos autores (Ring \& Vázquez, 1996) y que aparecieron en esta investigación, pertenecen a la categoría de Distracción; lo que los adolescentes hacen es dibujar, cantar, bailar, pasearse o distraerse. Por el contrario, un conjunto de estrategias a las que se les puede llamar Estrategias de Regulación Tecnológica, no han sido referidas por ninguna investigación y en éstas los adolescentes mencionan que una forma de afrontar la depresión es a través de ver la televisión, escuchar música o jugar video-juegos. Esta última es particularmente importante, ya que las investigaciones apuntan a una vinculación entre estas dos variables (jugar video juegos y depresión; Estallo, 1994). Sin embargo, será necesario estudiar más a fondo esta interacción para determinar el vínculo causa-efecto, es decir, los adolescentes se ensimisman en el juego por sentirse deprimidos y de esta forma lo afrontan; o por el contrario, el hecho de sumergirse en los videojuegos les provoca depresión.

Un tipo de estrategias mencionadas por ambos sexos, pero con mayor valor para las mujeres, fue un conjunto de comportamientos orientados hacia la búsqueda del apoyo social, es decir las Estrategias de Regulación Social, en las que se busca aliviar la depresión a partir del apoyo que pueden brindar tanto amigos como familiares. Es innegable el valor, tanto protector como causante, de la depresión (Valadez-Figueroa, AmezcuaFernández, Quintanilla-Montoya, \& GonzálezGallegos, 2005), que tienen estos grupos sociales y era de esperarse que emergieran como variables asociadas al fenómeno. Es de notarse que el orden en que aparecen es lo esperado para el caso de los adolescentes, es decir a quien se recurre en primer lugar es a los amigos y luego a los familiares, no es necesario que sean los padres (Leyva-Jiménez, Hernández-Juárez, Nava-Jiménez, \& López Gaona, 2007). 
También se observó que los adolescentes presentaronEstrategias de RegulaciónEmocional, donde se pretende afrontar la depresión a partir del control o manifestación de las emociones; pueden ser socialmente aceptadas como el ser feliz, amar o sonreír, o inaceptables como el enojo, el coraje, la rabia o el gritar. En ese sentido Lazarus y Folkman (1986) plantean que este tipo de estrategias no resuelven el problema.

Una última categoría de estrategias fueron las Estrategias de Regulación Cognitiva, se les nombra de esta forma porque las actividades que realizan están orientadas hacia un ejercicio mental como lo es el pensar, leer o escribir. Quizá el inconveniente de este tipo de estrategias es el ensimismamiento y el soliloquio en el que pueden caer los adolescentes y que a la postre los lleve a cometer el suicidio (Jiménez, Mondragón, \& González, 2007; Pliego, 2001). Por el contrario, si estas estrategias se aprovechan de manera adecuada el adolescente puede escribir lo que siente, de tal forma que aquellos que estén cerca de él puedan conocer lo que le está ocurriendo y darle la atención adecuada.

Para finalizar, es importante puntualizar que al menos en los diversos conjuntos SAM presentados en esta investigación, la mayor parte de las estrategias que dicen utilizar los adolescentes en realidad no llegan a resolver el estado de ánimo deprimido, lo que puede contribuir a explicar porque la depresión se atiende cuando se es adulto (Medina-Mora \& cols. 2003). Es decir, las estrategias que emplean los adolescentes están funcionando como paliativos para el malestar depresivo, pueden dar cierta tranquilidad temporal pero no se soluciona el problema (Valdez-Medina, 2009). Por otro lado, al nombrar a las estrategias se ha empleado de manera deliberada el titulo de "Estrategia de Regulación..." y se coloca el nombre correspondiente, se ha hecho de esta forma porque la depresión es un equilibrio perdido (Valdez- Medina, 2009) y las estrategias de afrontamiento lo que hacen es regular para obtener la homeostasis perdida. 


\section{Referencias}

Ando, M. (2002). Relationship among mental health, coping styles and mood. Psychological Reports. 90, 606-612.

Benjet, C., Borges, G., Medina-Mora, M. E., FleizBautista, C., \& Zambrano-Ruiz, J. (2004). La depresión con inicio temprano: prevalencia, curso natural y latencia para buscar tratamiento. Salud Pública de México, 46(5), 417-424.

Boschetti-Fentanes, B. (2004). La depression y su manejo en el ámbito de la medicina familiar. Archivos en Medicina Familiar, 6(3), 61-63.

Cevallos-Rivera, J. J., Ochoa-Muñoz, J., \& CortezPérez, E. (2000). Depresión en adolescencia. Su relación con actividad deportiva y consumo de drogas. Revista Medica del Instituto Mexicano del Seguro Social, 38(5), 371-379.

Consejo Nacional Contra las Adicciones. (2000). Encuesta Nacional de Adicciones. México: Secretaria de Salud.

Consejo Nacional de Población. (2002). Situación actual de las y los jóvenes en México. Diagnóstico Sociodemográfico. México: CONAPO.

Craig, G. J. (1997). Desarrollo Psicológico. México: Prentice Hall.

Encuesta Nacional de la Juventud. (2000). Resultados Generales. México: Secretaria de Educación Pública, Instituto Mexicano de la Juventud.

Estallo, J. A. (1994). Video juegos, personalidad y conducta. Psicothema. 6, 181-190.

Figueroa, M. I., Contini, N., Lacuzana, A. B., Levín, M., \& Estévez, S. A. (2005). Las estrategias de afrontamiento y su relación con el bienestar psicológico. Un estudio con adolescentes de nivel socioeconómico bajo de Tucumán (Argentina). Anales de Psicología, 21(1), 6672.
Figueroa, J., González, E., \& Solís, V. (1981). Una aproximación al Problema del Significado: Las Redes semánticas. Revista Latinoamericana de Psicología, 13(3), 447-458.

Frydenberg, E., \& Lewis, R. (1997). ACS. Escalas de Afrontamiento para Adolescentes. Manual. Madrid: TEA.

Galaif, E. R., Sussman, S., Chou, C-P., \& Wills, T. A. (2003). Longitudinal relations among depresión, stress, and doping high risk youth. Journal of Youth and Adolescence, 32, 243258.

Gamboa, C. I. P., \& Flores, G. M. M. (2006). Asertividad, enfrentamiento y depresión en Adolescentes Yucatecos. La Psicología Social en México, 11, 110-115.

González, E. S., Cruz, U. D. P., \& Martínez, M. X. V. (2007). La medición de la depresión en adolescentes: una propuesta psicométrica. Psicología y Salud, 17(2), 199-205.

González, E. S., \& Valdez, M. J. L. (2005). Significado psicológico de la depresión en médicos y psicólogos. Psicología y Salud, 15(2), 257-262.

González-Forteza, C. (1992). Estrés psicosocial y respuestas de enfrentamiento: impacto sobre el estado emocional en adolescentes. Tesis de Maestría.UNAM.

González-Forteza, C., Andrade-Palos, P., \& Jiménez, A. (1997). Estresores cotidianos familiares, sintomatología depresiva e ideación suicida en adolescentes mexicanos. Acta Psiquiátrica y Psicológica de América Latina, 43(4), 319 -326.

González-Forteza, C. F., Pick, S., Collado, M. M. E., Álvarez, M., \& Jiménez, A. (1996). Estresores de la sexualidad de los adolescentes y su forma de enfrentarlos. Un estudio exploratorio en México. Acta Psiquiátrica y Psicológica de América Latina, 42(1), 43-49.

González-Forteza, C., Villatoro, J., Pick. S., \& Collado, M. E. (1998). Estrés psicosocial y su relación con las respuestas de enfrentamiento 
y el malestar emocional en una muestra representativa de adolescentes al sur de la ciudad de México: Análisis según nivel socioeconómico. Salud mental, 21(2), 37-45.

Haghitgou, H., \& Peterson, C. (2001). Coping and depressive symptoms among Iranian students. The Journal of Social Psychology, 135,175-180.

Hewitt, P. L., Flett, G. L., \& Endler, N. S. (1995). Perfectionism, Doping, and depression symptomatology in a Clinical Simple. Clinical Psychology and Psychotherapy, 2, 47-58.

Hussain, F. A., \& Cochrane, R. (2003). Living with depression: coping strategies used by south Asian women, living in the UK, suffering from depression. Mental Health, Religion and Culture, 6, 21-24.

Instituto Nacional de Estadística, Geografía e Informática (2000). Daños a la salud. Tabulados Básicos y por Entidad. Mexico: INEGI.

Jiménez, T. A., Mondragón, B. L., \& González, F. C. (2007). Selfesteem, depressive symtomatology, and suicidal ideation in adolescents: results of three studies. Salud Mental, 30, 20 - 26.

Kuyken, W., \& Brewin, C. R. (1994). Stress and Coping in Depressed women. Cognitive Therapy and Research, 18, 403-412.

Lazarus, R. S., \& Folkman, S. (1986). Estrés y procesos cognitivos. Barcelona: Martínez Roca.

Leyva-Jiménez, R., Hernández-Juárez, A. M., Nava-Jiménez, G., \& López-Gaona, V. (2007). Depresión en adolescentes y funcionamiento familiar. Revista Medica del Instituto Mexicano del Seguro Social, 45(3), 225-232.

Mariño, M. C., Medina-Mora, M. E., Chaparro, J.J., \& González-Forteza, C. (1993). Confiabilidad y estructura factorial del CES-D en una muestra de adolescentes mexicanos. Revista Mexicana de Psicología, 10(2), 141-146.
Marusic, A., \& Goodwin, R. D. (2006). Suicidal and deliberate Self-Harm Ideation Among Parents with Psysical Illnes: The Role of Copyng Styles. Journal of Suicide and LiveThreatening Behavior, 36(3), 323-328.

McWilliams, L. A., Cox, B. J., \& Enns, M. W. (2003). Use of the Coping Inventory and Stressful situations in a clinical depressed sample. Facture Structure, Personality corelates and prediction of distress. Journal of Clinical Psychology, 59(12), 1371-1385.

Medina-Mora, M. E., Borges, G., Lara Muñoz, C., Benjet, C., Blanco Jaimes, J., Fleiz Bautista, C., Villatoro Velázquez, J., Rojas Guiot, E., Zambrano Ruíz, J., Casanova Rodas, L., \& Aguilar Gaxiola, S. (2003). Prevalencia de trastornos mentales y uso de servicios: Resultados de la Encuesta Nacional de Epidemiología Psiquiátrica en México. Salud Mental, 26(4), 1-16.

Meyer, M. (2007). Estilos de afrontamiento en situaciones de tristeza en estudiantes de la Universidad Iberoamericana mediante el Coping Inventory of Depression (CID). Psicología Iberoamericana, 15(1), 24-33.

Papalia, D. (2004). Psicología del Desarrollo. México: McGraw-Hill.

Pardo, A. G., Sandoval, D. A., \& Umbarila, Z. D. (2004). Adolescencia y depresión. Revista Colombiana de Psicología, 13, 13-28.

Pliego, A. (2001). Cómo detectar y vencer la depresión en jóvenes. México: Selector.

Ring, J., \& Vázquez, C. (1996). Estrategias de afrontamiento ante la depresión: un análisis de su frecuencia y utilidad mediante el Doping Inventory of depresión. Psicología Conductual, 4, 9-28.

Santos-Preciado, B. J. I., Villa-Barragan, J. P., García-Avilés, M. A., León-Álvarez, G. L., Quezada-Bolaños, S., \& Tapia-Conyer, R. (2003). La transición epidemiológica de los y las adolescentes en México. Salud Pública de México, 45(1), 140-S152. 
Shaffer, D. R. (2000). Psicología del Desarrollo: infancia y adolescencia. México: International Thomson Editores.

Sheung-tak, Ch., \& Chan, A. C. M. (2007). Pathaways from stress to suicidality and the protective effect of social support in Hong Kong Adolescents. Journal of Suicide and Life Threatening Behavior, 37(2), 187-196.

Smith, M., Wethington, E., \& Zhan, G. (1996). Self-concept clarity and preferred coping styles. Journal of Personality, 64(2), 407434.

Valadez-Figueroa, I., Amezcua-Fernández, R., Quintanilla-Montoya, R., \& GonzálezGallegos, N. (2005). Familia e Intento suicida en el adolescente de educación media superior. Archivos en Medicina Familiar, 7(3), 69-78.

Valdez-Medina, J. L. (1998). Las redes semánticas, Usos y Aplicaciones en Psicología Social. México: Universidad Autónoma del Estado de México

Valdez, M. J. L. (2007). Hombres y Mujeres en México, dos mundos distintos pero complementarios. México: Universidad Autónoma del estado de México.

Valdez, Medina. J. L. (2009). Teoría de la Paz o Equilibrio: una nueva teoría que explica las causas del miedo y del sufrimiento, y que nos enseña a combatirlos. México: EDAMEX.

Valdez-Medina, J. L., \& Hernández, G. A. (1986). El significado psicológico de Padre, madre, Dios y Virgen: Redes semánticas, Convergencias conceptuales. Tesis de Licenciatura. México: Universidad Autónoma de México.

Whatley, S. L., Foreman, A. C., \& Richards, S. (1998). The relationship of coping style to dysphoria, anxiety, and anger. Psychological Reports, 83, 783-791. 